EPJ Web of Conferences 59, 06003 (2013)

DOI: $10.1051 /$ epjconf/20135906003

(C) Owned by the authors, published by EDP Sciences, 2013

\title{
Study of shockwave method for diagnosing the radiation fields of laser-driven gold hohlraums
}

\author{
Yongsheng Li ${ }^{1,2, a}$, Ke Lan ${ }^{1}$, Wenyi Huo ${ }^{1}$, Dongxian Lai ${ }^{1}$, Yaoming Gao ${ }^{1}$ \\ and Wenbing $\mathrm{Pei}^{1}$ \\ 1 Institute of Applied Physics and Computational Mathematics, 100094 Beijing, China \\ 2 The Graduate School of China Academy of Engineering Physics, 100088 Beijing, China
}

\begin{abstract}
Besides the routinely used broad-band x-ray spectrometer (Dante or SXS), ablative shockwave method is often used to diagnose the radiation fields of laser-driven Hohlraums. The x-ray ablation process of Aluminum and Titanium is studied numerically with a 1-D radiation hydrodynamic code RDMG [F. Tinggui et al., Chin. J. Comput. Phys. 16, 199 (1999)], based on which a new scaling relation of the equivalent radiation temperature with the ablative shock velocity in Aluminum plates is proposed, and a novel method is developed for determining simultaneously the radiation temperature and the M-band (2-4 keV) fraction in laser-driven gold Hohlraums.
\end{abstract}

\section{INTRODUCTION}

For laser-indirect-drive inertial confinement fusion (ICF) with the so called central-hotspot-igniting scheme, the radiation fields of high-Z cavity (or hohlraums) driven by high power laser facilities such as NIF (National Ignition Facility) and LMJ (Laser Mega-Joule), must be carefully designed and controlled to ignite and burn the DT fuels contained in a little $(\sim 1 \mathrm{~mm}$ diameter $)$ plastic capsule, which is in turn placed in the centre of high-Z cavities. Firstly, to achieve an implosion velocity high enough to ignite DT capsule, the radiation temperature should be about $\sim 300 \mathrm{eV}$. Secondly, the temporal profile of hohlraums radiation fields should be precisely shaped to launch multiple shocks for compressing the main fuel quasi-isentropically, therefore to reduce the total laser energy needed for ignition. Lastly, to reduce the hydro-instabilities at the fuel-ablator interface, and to lower the adiabat of adjacent DT fuel, high energy x rays (specifically, the Au M-band radiation emitted from laser spots) must be precluded from preheating the ablator materials near the fuel by mid- / high- $Z$ dopants in ablator. So, accurate measurements of the peak temperature, the pulse shape and the high energy x-ray amount of the hohlraums radiation fields are of great importance. Currently, there are two independent experimental approaches [1-3] to diagnose the hohlraums radiation field: (1) direct measurement of the radiation flux with broad-band X-ray spectrometer (Dante [4] or SXS [5] and (2) observing the velocity $D_{\mathrm{S}}$ of the shock wave in an $\mathrm{Al}$ witness plate driven by hohlraums radiation field, where the radiation temperature is inferred via a scaling relation [2] of $T_{\mathrm{R}}$ with $D_{\mathrm{s}}$.

What we concerned with in this proceeding is the second method. The x-ray ablation process of mid$\mathrm{Z}$ materials, such as Aluminum and Titanium, is studied numerically with a 1-D radiation hydrodynamic code RDMG [6]. It is found that the ablative shock velocity in Aluminum depends not only on the peak radiation-temperature but also on the pulse shape and the spectrum (specifically, Au M-band fraction). With the postulation of equilibrium radiation sources, a scaling relation of peak radiation temperatures

\footnotetext{
ae-mail: li_yongsheng@iapcm.ac.cn
}

This is an Open Access article distributed under the terms of the Creative Commons Attribution License 2.0, which permits unrestricted use, distribution, and reproduction in any medium, provided the original work is properly cited. 

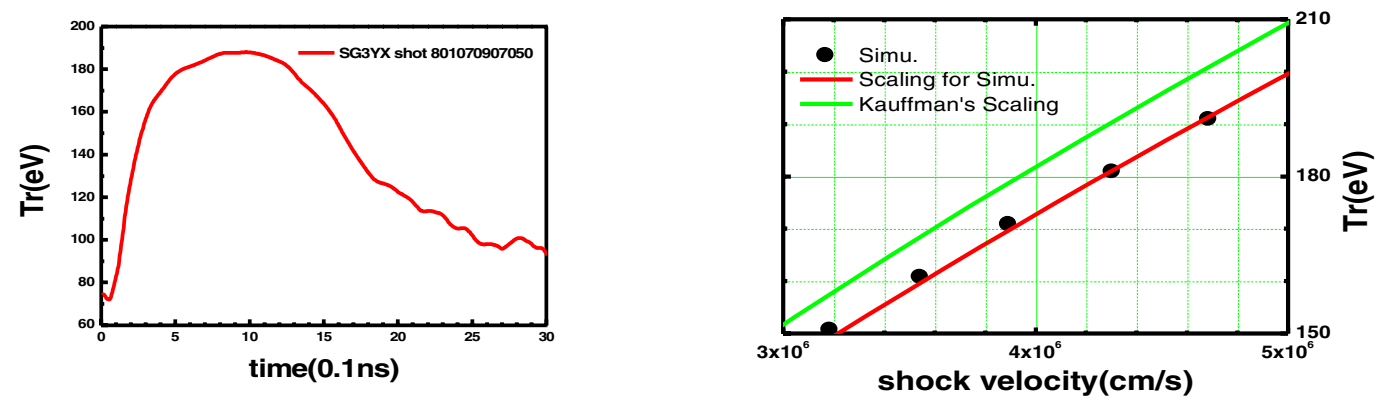

Figure 1. $x$-ray source of hohlraum driven by 1 ns square laser pulse on SGIII prototype (left) and the scaling relations of $T_{\mathrm{R}}$ with $D_{\mathrm{s}}$ (right).

with $\mathrm{Al}$ shock velocities is proposed, being applicable to $1 \mathrm{~ns}$ pulse laser-driven hohlraums, and the dependence on the pulse shape being negligible. Further a novel method is proposed, based on our study on the responses of ablative shock waves in Aluminum and Titanium to Au M-band flux, for determining the $\mathrm{Au} \mathrm{M}$-band (2-4 keV) fraction of X-ray sources, which provides a complementary means for diagnosing the M-band flux in laser-driven gold hohlraums.

\section{EFFECTS OF PULSE SHAPE OF X-RAY SOURCE}

For strong shock waves, the velocity $D_{\mathrm{s}}$ scales as $\sim P^{1 / 2}$, and according to a steady ablation model [7] for low-Z materials, the radiative ablation pressure $P$ scales as $\sim T_{\mathrm{R}}^{3.5}$. So it can be derived that $T_{\mathrm{R}} \sim D_{\mathrm{s}}^{4 / 7}$. Considering the influence of the equation of state (EOS) and reradiation of Aluminum, the radiative ablation process is no longer stationary, but a similar relation may be expressed as $T_{\mathrm{R}}=\alpha \times D_{\mathrm{s}}^{\beta}$, where the parameters $\alpha$ and $\beta$ can be determined via numerical simulation and fitting. Kauffman et al. [2] proposed the following scaling relation

$$
T_{\mathrm{R}}=0.0126 \times D_{\mathrm{s}}^{0.63},
$$

and it was believed that $\alpha$ was determined by the EOS and $\beta$ by the reradiation of Aluminum. Yet that is not the whole story. We simulated the ablation process of Aluminum plates using the X-ray source of the Hohlraums driven by $1 \mathrm{~ns}$ square laser pulse on SG III Prototype, as shown in Fig. 1 (left). The simulated shock velocity at a given peak radiation temperature is faster than that derived from formula (1), as shown in the right figure of Fig. 1. Fitting our simulation results can produce a new scaling relation

$$
T_{\mathrm{R}}=0.0092 \times D_{\mathrm{s}}^{0.647},
$$

which is about $10 \mathrm{eV}$ lower than formula (1) at a given shock velocity of $(4-8) \times 10^{6} \mathrm{~cm} / \mathrm{s}$, as shown with red line in the right figure of Fig. 1. Detailed analysis [8] show that, compared to the X-ray source shown in the left figure of Fig. 1, the prepulse of the PS22 x-ray source on NOVA (Fig. 4 in Ref. [2]) used to derive formula (1) ablates more Aluminum plasmas before the arrival of the main pulse, which absorbs and reemits backward more x-ray flux, therefore little x-ray flux arrives at the ablation front and produces a smaller ablation pressure and a slower shock wave. That means the scaling relation of the peak radiation temperature $T_{\mathrm{R}}$ with the ablation shock velocity $D_{\mathrm{s}}$ in Aluminum plates can be affected, to some extent, by the temporal profile of x-ray sources, i.e. there are no universal scaling relations. Note that the scaling relation (2) is only applicable to the hohlraums powered by $1 \mathrm{~ns}$ square laser pulse. 

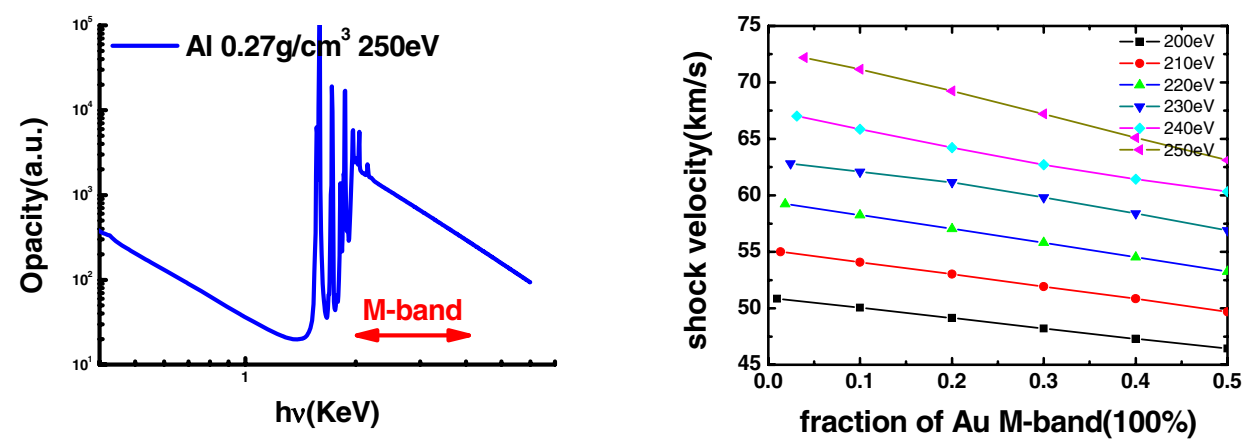

Figure 2. Opacity of Aluminum plasmas (left) and shock velocties vs. M-band fraction (right).


Figure 3. Opacity of Titanium plasmas (left) and shock velocties vs. M-band fraction (right).

\section{INFLUENCE OF AU M-BAND FLUX ON SHOCK VELOCITY}

Not only the temporal profile, but also the spectrum of x-ray sources can affect the ablation shock velocities in Aluminum plates. As shown in Fig. 2 (left), the opacity of Aluminum plasmas varies with frequency, and is much higher within the Au M-band ( $2 \sim 4 \mathrm{keV})$ than within $400 \mathrm{eV} \sim 1 \mathrm{keV}$. Ascribed to this property, the shock velocity in Aluminum driven by an $\mathrm{x}$-ray source with a higher fraction of $\mathrm{Au}$ M-band flux will be slower than that driven by an x-ray source of the same intensity but with a lower fraction of Au M-band flux. We have performed lots of simulations of the x-ray ablation process of Aluminum plates using RDMG, with the peak radiation temperatures varying from $200 \mathrm{eV}$ to $250 \mathrm{eV}$. By increasing the Au M-band fraction $f_{\mathrm{M}}$ in the $\mathrm{x}$-ray source, the shock velocity decreases monotonically, as shown in Fig. 2 (right).

On the contrary, the Titanium opacity and the response of its ablation shock velocity to the $\mathrm{Au}$ M-band fraction of x-ray sources are quite different from those of Aluminum. Fig. 3 (left) shows the opacity of Titanium, the opacity within Au M-band is quite smaller than within $400 \mathrm{eV} \sim 1 \mathrm{keV}$, and the shock velocity increases with the increasing of M-band fraction in x-ray sources. Since the Au M-band fraction can apparently influence the shock velocities in Aluminum and Titanium, they can not be solely used to determine the peak radiation temperatures of high-power-laser driven gold hohlraums, where the Au M-band flux contributes a high enough fraction of x-ray flux. But due to their contrary opacity properties, they can be used jointly to determine the peak radiation temperature and M-band fraction simultaneously, which will be detailed in next section. 


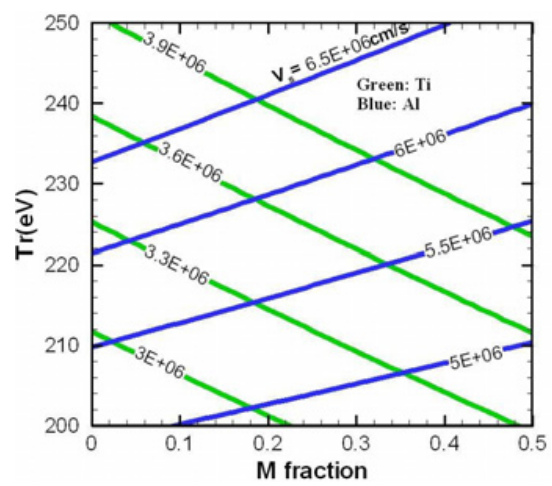

Figure 4. Shock velocity contours in $T_{\mathrm{R}}-f_{\mathrm{M}}$ plane for Aluminum (blue line) and Titanium (green line).

\section{AL/TI SHOCKWAVES TO DETERMINE $\mathrm{F}_{\mathrm{M}}$ AND $\mathrm{T}_{\mathrm{R}}$ SIMULTANEOUSLY}

As has been detailed above, for x-ray sources with a higher fraction of Au M-band fraction, both the temporal profile and the Au M-band fraction can apparently affect the shock velocities of Aluminum and Titanium. The scaling relations of $T_{\mathrm{R}}$ with $D_{\mathrm{s}}$ and $f_{\mathrm{M}}$ can be expressed with the following formula $T_{\mathrm{R}}=\alpha \times D_{\mathrm{s}}^{\beta}$, where $\alpha=a \times b^{f_{M}}$ and $\beta=c+d \times f_{M}$. By fitting our simulation results shown in Fig. 2 and Fig. 3, we can obtain that $\alpha=0.01379 \times 0.0508^{f_{M}}, \beta=0.6205+0.2012 f_{M}$ for Al and $\alpha=0.01267 \times 0.2671^{f_{M}}, \beta=0.6520+0.07156 f_{M}$ for Ti. If the shock velocities of $\mathrm{Al}$ and of Ti can be obtained in one shot, we can draw out the velocity contours of the two materials in a $T_{R^{-}} f_{M}$ plane. The cross point tells us, simultaneously, the peak radiation temperature and Au M-band fraction of the laser-driven gold hohlraums. More details can be found in Ref. [9].

\section{SUMMARY}

The scaling relation of $T_{\mathrm{R}}$ with $\mathrm{Al}$ shock velocity depends on the temporal profile of $\mathrm{x}$-ray source. Or, there are no universal scaling relations. For the cases where the M-band fraction in $\mathrm{x}$-ray sources is higher enough, the velocities of $\mathrm{Al}$ and $\mathrm{Ti}$ ablation shocks depend on both the total incident flux and the M-band fraction of it. A new method is proposed to diagnose the peak radiation temperature and M-band fraction simultaneously.

This work was partially supported by the National Natural Science Foundation of China under Grant No. 11105014.

\section{References}

[1] J. D. Lindl, Phys. Plasmas 2, 3933 (1995); J. D. Lindl, P. Amendt, R. L. Berger, S. G. Glendinning, S. H. Glenzer, S. W. Haan, R. L. Kauffman, O. L. Landen, and L. J. Suter, Phys. Plasmas 11, 339 (2004)

[2] R. L. Kauffman, L. J. Suter, C. B. Darrow, J. D. Kilkenny, H. N. Kornblum, D. S. Montgomery, D. W. Phillion, M. D. Rosen, A. R. Theissen, R. J. Wallace, and F. Ze, Phys. Rev. Lett. 73, 2320 (1994) 
[3] R. E. Olson, D. K. Bradley, G. A. Rochau, G. W. Collins, R. J. Leeper, and L. J. Suter, Rev. Sci. Instrum. 77, 10E523 (2006)

[4] H. N. Kornblum, R. L. Kauffman, and J. A. Smith, Rev. Sci. Instrum. 57, 2179 (1986)

[5] K. Sun, J. Yang, and Z. Zheng, High Power Laser and Partical Beams 2, 16 (1990) (in Chinese)

[6] T. Feng, D. Lai, and Y. Xu, Chin. J. Comput. Phys. 16, 199 (1999) (in Chinese)

[7] S. P. Hatchett, Ablation Gas Dynamics of Low-Z Materials Illuminated by Soft X-rays (Lawrence Livermore National Laboratory, Livermore, 1991)

[8] Y. Li, K. Lan, D. Lai, Y. Gao and W. Pei, Phys. Plasmas 17, 042704 (2010)

[9] Y. Li, W. Huo and K. Lan, Phys. Plasmas 18, 022701 (2011) 